\title{
Análise de prescrições em unidades de terapia intensiva em um hospital público da região norte do Brasil
}

\author{
Analysis of prescriptions in intensive care units in a public hospital in the north region of Brazil \\ Análisis de recetas en unidades de cuidados intensivos en un hospital público de la región norte de
}

Brasil

Recebido: 07/12/2021 | Revisado: 15/12/2021 | Aceito: 17/12/2021 | Publicado: 22/12/2021

\author{
Valcione da Silva Gretzler \\ ORCID: https://orcid.org/0000-0001-9808-7201 \\ Hospital Regional de Cacoal, Brasil \\ E-mail:valcionegretzler18@gmail.com \\ Elissa Gonçalves de Oliveira e Silva \\ ORCID: https://orcid.org/0000-0001-8022-4511 \\ Hospital Regional de Cacoal, Brasil \\ E-mail:elissas_br@yahoo.com.br \\ Thais Dias Zumack \\ ORCID: https://orcid.org/0000-0002-5356-4956 \\ Hospital Regional de Cacoal, Brasil \\ E-mail: thais_zumack93@hotmail.com \\ Danielle Maesima Saito \\ ORCID: https://orcid.org/0000-0001-7735-0066 \\ Hospital Regional de Cacoal, Brasil \\ E-mail:danisaito30@hotmail.com \\ Simone Rosária Soares de Moraes Cunha \\ ORCID: https://orcid.org/0000-0002-1105-7868 \\ Hospital Regional de Cacoal, Brasil \\ E-mail:simone.rsmc@gmail.com \\ Francisca Letícia Moreira Lustosa Portela \\ ORCID: https://orcid.org/0000-0002-6053-8115 \\ Hospital Regional de Cacoal, Brasil \\ E-mail:leticiaportella@gmail.com \\ Welliny Mariana Posse Fuzari \\ ORCID: https://orcid.org/0000-0001-7951-7317 \\ Central Farma, Brasil \\ E-mail: wellinypossefuzari@gmail.com
}

\begin{abstract}
Resumo
O presente estudo tratou de analisar um conjunto de prescrições em duas unidades de terapia intensiva adulto em um hospital público da região norte do Brasil. Realizada uma pesquisa transversal, quantitativa, documental e descritiva, considerando 270 prescrições de forma. A análise foi realizada por um profissional farmacêutico apto, utilizando como instrumento para a coleta de dados um questionário com dezessete questões assertivas. Esta pesquisa foi realizada em unidades de terapia intensiva adulto com um total de dezoito leitos no período de 1 a 15 de abril do ano de 2021. Nesta pesquisa foram identificadas divergências nos indicadores: peso do paciente, número do prontuário, posologia, aprazamento, Denominação Comum Brasileira, forma de diluição, velocidade de infusão e a utilização da expressão se necessário. Os erros de prescrições se tornaram comuns atualmente, sendo evidenciada como risco a saúde e a vida dos pacientes. Considerando o índice de divergência em prescrições faz-se necessário a atuação de forma integral do profissional farmacêutico para a análise de prescrições e intervenções farmacêuticas, buscando assim garantir uma melhor farmacoterapia aos pacientes.
\end{abstract}

Palavras-chave: Unidade de Terapia Intensiva; Farmácia hospitalar; Segurança do paciente.

\begin{abstract}
The present study analyzed a set of prescriptions in two adult intensive care units in a public hospital in northern Brazil. A cross-sectional, quantitative, documentary and descriptive research was carried out, considering 270 shape prescriptions. The analysis was performed by a qualified pharmacist, using a questionnaire with seventeen assertive questions as an instrument for data collection. This research was carried out in adult intensive care units with a total of
\end{abstract}


eighteen beds in the period from April 1st to 15th, 2021. In this research, divergences in the indicators were identified: patient weight, medical record number, dosage, schedule, Denomination Common Brazilian, form of dilution, infusion rate and use of the expression if necessary. Prescription errors have become common nowadays, being evidenced as a risk to the health and life of patients. Considering the divergence index in prescriptions, it is necessary for the pharmacist to work in an integral way for the analysis of prescriptions and pharmaceutical interventions, thus seeking to ensure a better pharmacotherapy for patients.

Keywords: Intensive Care Unit; Hospital pharmacy; Patient safety.

\section{Resumen}

El presente estudio analizó un conjunto de prescripciones en dos unidades de cuidados intensivos para adultos de un hospital público del norte de Brasil. Se realizó una investigación transversal, cuantitativa, documental y descriptiva, considerando 270 prescripciones de forma. El análisis fue realizado por un farmacéutico calificado, utilizando un cuestionario con diecisiete preguntas asertivas como instrumento para la recolección de datos. Esta investigación se realizó en unidades de cuidados intensivos para adultos con un total de dieciocho camas en el período del 1 al 15 de abril de 2021. En esta investigación se identificaron divergencias en los indicadores: peso del paciente, número de historia clínica, posología, horario, Denominación Brasileño común, forma de dilución, velocidad de infusión y uso de la expresión si es necesario. Los errores de prescripción se han vuelto comunes en la actualidad, evidenciándose como un riesgo para la salud y la vida de los pacientes. Considerando el índice de divergencia en las prescripciones, es necesario que el farmacéutico trabaje de manera integral en el análisis de prescripciones e intervenciones farmacéuticas, buscando así asegurar una mejor farmacoterapia para los pacientes.

Palabras clave: Unidad de Cuidados Intensivos; Farmacia hospitalaria; Seguridad del paciente.

\section{Introdução}

Uma das principais ferramentas utilizadas no processo do cuidado ao paciente e o ponto inicial para a utilização de medicamentos é a prescrição médica. Seu trajeto é uma atividade complexa e multiprofissional, que envolve médicos, farmacêuticos, enfermeiros, técnicos e auxiliares. (Neri et al., 2011). Este processo complexo vai desde a prescrição até a administração e monitoramento das reações do medicamento. (Brasil, 2013).

Os erros de medicamentos infelizmente são considerados comuns, e podem ocasionar danos severos a saúde dos pacientes. Dentre os erros correlacionados a medicamentos, os mais comuns se dão no momento da prescrição, sendo estes considerados os mais complexos (Cunha, 2018). No ano de 2004 a Organização Mundial da Saúde (OMS) visando diminuir os erros e garantir a segurança dos pacientes, lançou um programa o qual tem como intuito uma aliança mundial para a segurança do paciente. Este programa é composto por diversos países como Brasil e Canadá que visam buscar medidas para assegurar a qualidade da assistência prestada, como a implantação de protocolos como por exemplo pratica de higiene das mãos, uso e administração de medicamentos (Brasil, 2014). E em abril do ano de 2013 foi criada a Portaria MS/GM nº 529, que tem como objetivo contribuir para a qualificação do cuidado em saúde nos estabelecimentos de saúde (Brasil, 2013).

No ano de 2015, foi realizada uma pesquisa pela faculdade de Medicina da Universidade Federal de Minas Gerais (UFMG) e pelo Instituto de Estudos de Saúde Suplementar (IESS), que mostrou que eventos adversos - erros, negligência ou baixa qualidade do serviço - podem ser a primeira ou segunda causa de óbitos no país, à frente de doenças cardiovasculares ou câncer, que mataram 339.672 e 196.954 em 2013, respectivamente. Estes eventos adversos representam problemas que vão desde erros de dosagem de medicamentos ou aplicação de medicamentos, até uso incorreto de equipamentos e infecção hospitalar, estas falhas acarretaram em 434.000 óbitos, o equivalente a 1.000 mortes por dia (Couto et al., 2017).

Desde 1999 o Institute of Medicine dos Estados Unidos, com a publicação To Err Is Human: Building a Safer Health System, buscou aumentar a conscientização sobre erros de medicamentos e incentivou ações governamentais para a prevenção desses eventos. Já em 2007 o mesmo instituto declarou que o nível desses eventos adversos e as consequências são inaceitáveis (Tânia et al., 2010). É necessário frisar que erros de medicamentos são considerados de causas multifatoriais, e dentre as 
principais causas, destacam a falta de conhecimento do fármaco, falha de interpretação, violação dos protocolos, falta de informações sobre o paciente entre outros (Pena, 2015).

Existem diversos itens necessários para garantir a segurança do paciente, seguindo os cumprimentos de procedimento legais e protocolos dos procedimentos institucionais, podemos citar desde a identificação do paciente que deve ser realizada de forma legível, com o nome completo, data de nascimento, número do leito e da clínica ou UTI - Unidade de Terapia Intensiva, em que o paciente se encontra, número do prontuário, além disso deve conter a identificação do prescritor com nome e número do registro do conselho de medicina e assinatura do mesmo; os medicamentos prescritos devem seguir a Denominação Comum Brasileira (DCB), além da indicação de alergias, dose, concentração, posologia, diluição, via de administração, tempo de infusão e velocidade de infusão corretos (Brasil, 2017).

A atuação da equipe multiprofissional é essencial para garantir a diminuição desses erros, principalmente dos profissionais farmacêuticos, médicos e enfermeiros, pois os mesmos estão ligados de forma direta no que tange ao tratamento farmacoterapêutico do paciente (Amaro, 2017; Alvim, 2015). Dentre estes profissionais, o farmacêutico se destaca tendo como dever analisar de forma criteriosa cada prescrição e transmitir conhecimento a equipe multiprofissional sobre o tratamento farmacoterapêutico do paciente, através de análises e intervenções (De Lima, 2013).

Um dos métodos utilizados com frequência em diversas unidades hospitalares é o emprego dos 13 certos. Este método se mostrou eficaz, pois o mesmo exemplifica a base para a administração de fármacos, que consiste em: prescrição certa, paciente certo, validação certa, medicamento certo, forma farmacêutica correta, dose certa, horário correto, compatibilidade, via de administração certa, tempo de infusão do medicamento, ação certa, orientação do paciente e registro certo (CRE-SP, 2017; Marquins, 2015).

A atuação do farmacêutico clínico se torna essencial para garantir a diminuição destes erros, pois no momento da validação da prescrição este profissional consegue visualizar erros, e a realização de notificação de cunho orientativo se torna de extrema importância para ampliar o conhecimento dos índices e das gravidades dos eventos relacionados aos erros de medicamentos (Costa, 2017; Melo, 2017). Devido a estes fatores, essa pesquisa tem como objetivo caracterizar as prescrições de uma unidade de terapia intensiva na região norte do Brasil.

\section{Metodologia}

Esta pesquisa é caracterizada como transversal, quantitativa, documental e descritiva, denominada "análise de prescrições em uma unidade de terapia intensiva em um hospital público da região norte do Brasil”, realizada em duas unidades de terapia intensiva, totalizando 18 leitos. A amostra utilizada para a pesquisa foram prescrições médicas, da primeira quinzena do mês de abril do ano de 2021, totalizando 270 prescrições médicas.

A pesquisa respeitou os aspectos éticos presentes na Resolução No .4 .594 .742 do Conselho Nacional de Saúde e foi aprovada pelo Comitê de Ética sob o número CAAE: 43738621.2.0000.52984.

O instrumento utilizado para a coleta de dados teve como base um questionário adaptado do autor (Pires et al., 2017). Este questionário foi composto por dezessete questões objetivas, que avaliam a identificação do paciente e possíveis erros correlacionados aos medicamentos.

Os dados obtidos após a análises das prescrições foram organizados em formas de tabelas no programa Microsoft Office Excel 2013 e Microsoft Word 2013, com o auxílio de alguns mecanismos como frequência absoluta e mediana, e para melhor compreensão os dados foram divididos em duas tabelas. 


\section{Resultados e Discussão}

Para a elaboração desta pesquisa foram analisadas 270 prescrições médicas em unidade de terapia intensiva, na primeira quinzena do mês de abril do ano de 2021. Os resultados foram divididos para maior compreensão em duas tabelas: Tabela 1 - foram analisados os indicadores relacionados à identificação do paciente, e na Tabela 2 - foram analisados os indicadores relacionados aos medicamentos. A análise se deu mediante o cumprimento dos procedimentos legais e institucionais para a elaboração da prescrição médica, que são de extrema importância, pois a prescrição é considerada o principal instrumento de comunicação entre a equipe multiprofissional no cuidado ao paciente. Deve ser realizada com responsabilidade e conhecimentos técnicos, alinhada aos protocolos assistenciais instituídos afim de evitar erros. Sendo observado que a ausência de variáveis básicas numa prescrição médica pode acarretar em danos graves aos pacientes e também aos colaboradores da equipe.

Quanto aos componentes legais e os necessários para identificação dos pacientes constatou que todas as prescrições analisadas continham nome do paciente completo, nome da mãe correto, data de nascimento, data da prescrição, identificação do prescritor, seguindo as orientações do Ministério da Saúde e os protocolos hospitalares, sendo que os mesmos descrevem como de suma importância a necessidade dos itens acima supracitados, visando assim garantir a segurança dos pacientes. Porém foram constatadas algumas divergências nos indicadores peso do paciente e número do prontuário, onde 88 não informavam o peso do paciente e 21 não possuíam o número do prontuário (Tabela 1).

Alguns medicamentos são considerados doses dependentes do peso, e para melhor acompanhamento farmacoterapêutico é de fundamental importância a descrição do peso do paciente na prescrição, para que seja possível garantir a quantidade de fármaco e calcular o clearence de cada um, visando assim reduzir desperdícios e buscando a melhor terapia. Alguns fármacos não atingirão o limiar terapêutico adequado, devido à subdose, e em doses maiores que o permitido de acordo com o limiar terapêutico, podem provocar danos aos pacientes. Exemplo disso seriam as doses de sedativos utilizadas em UTI’s que em alguns momentos acabam ultrapassando esse limiar, dificultando o despertar do paciente e assim aumentando seu tempo de permanência no hospital, além do custo hospitalar. Também podemos citar os fármacos utilizados para tromboprofilaxia, essencial para os pacientes que permanecem restritos ao leito, rotina comum dos pacientes de UTI. Estes fármacos, caso utilizados acima do recomendado podem causar sangramentos graves, podendo levar a um desfecho não favorável ao paciente (Nacun, 2009; Cardinal, 2014).

Tabela 1: Critérios de identificação.

\begin{tabular}{|c|c|c|c|}
\hline INDICADORES & $\begin{array}{c}\text { Informações } \\
\text { Corretas }\end{array}$ & $\begin{array}{c}\text { Informações } \\
\text { Incoerentes }\end{array}$ & $\begin{array}{c}\text { Valor Absoluto da } \\
\text { amostra }\end{array}$ \\
\hline O nome do paciente está completo? & 270 & 0 & 270 \\
\hline O nome da mãe esta correto? & 270 & 0 & 270 \\
\hline A data de nascimento do paciente está correta? & 270 & 0 & 270 \\
\hline O peso do paciente esta na prescrição? & 182 & 88 & 270 \\
\hline O número do prontuário está na prescrição? & 249 & 21 & 270 \\
\hline A data da prescrição está correta? & 270 & 0 & 270 \\
\hline A prescrição está assinada? & 270 & 0 & \\
\hline
\end{tabular}


$\mathrm{Na}$ Tabela 2, foi realizada uma análise de forma integral dos indicadores: posologia dos medicamentos prescritos, via de administração, forma farmacêutica, aprazamento dos medicamentos, Denominação Comum Brasileira, diluição do fármaco, velocidade de infusão e foi verificado também a frequência e existência de informações como medicamentos de uso contínuo e medicamentos se necessário.

Tabela 2: Caracterização da prevalência dos principais tipos de erros.

\begin{tabular}{|c|c|c|c|c|c|c|c|}
\hline \multirow{2}{*}{ INDICADORES } & \multirow{2}{*}{$\begin{array}{c}\text { SIM } \\
\mathrm{n}=18\end{array}$} & \multirow{2}{*}{$\begin{array}{l}\text { NÃO } \\
\mathrm{n}=18\end{array}$} & \multirow{2}{*}{$\begin{array}{c}\text { NSA } \\
\mathrm{n}=18\end{array}$} & \multicolumn{3}{|c|}{ MEDLANA } & \multirow{2}{*}{$\begin{array}{c}\text { Valor Absoluto da } \\
\text { amostra }\end{array}$} \\
\hline & & & & SIM & NÃO & NSA & \\
\hline A posologia está correta? & $2415(72,68 \%)$ & $100(3,02 \%)$ & $808(24,32 \%)$ & 142 & 0 & 66 & 3323 \\
\hline A via de administração está correta? & $3628(99,23 \%)$ & $0(0 \%)$ & $0(0 \%)$ & 200 & 0 & 0 & 3656 \\
\hline $\begin{array}{l}\text { A via prescrita está de acordo com a forma } \\
\text { farmacêutica de escolha? }\end{array}$ & $3688(99,97 \%)$ & $1(0,03 \%)$ & $0(0 \%)$ & 206 & 0 & 0 & 3689 \\
\hline O aprazamento está correto? & $2158(63,71 \%)$ & $177(5,1 \%)$ & $1082(32,01 \%)$ & 130 & 73 & 0 & 3387 \\
\hline $\begin{array}{l}\text { O nome do fármaco está de acordo com a } \\
\text { Denominação comum Brasileira? }\end{array}$ & $2873(76,61 \%)$ & $0(0 \%)$ & $55(25,11 \%)$ & 164 & 55 & 0 & 3750 \\
\hline A forma de diluição está correta? & $1570(63,54 \%)$ & $0(0 \%)$ & $901(36,46 \%)$ & 85 & 0 & 42 & 2471 \\
\hline Está descrita a forma de diluição? & $1570(64,88 \%)$ & $77(2,98 \%)$ & $773(32,01 \%)$ & 77 & 77 & 34 & 2420 \\
\hline Contém informações da velocidade de infusão? & $698(19,19 \%)$ & $200(5,5 \%)$ & $158(74,18 \%)$ & 45 & 158 & 10 & 3637 \\
\hline Usa a expressão uso contínuo? & $0(0 \%)$ & $0(0 \%)$ & - & 0 & 0 & 0 & 0 \\
\hline Utiliza a expressão se necessário? & $514(100 \%)$ & $0(0 \%)$ & $0(0 \%)$ & 30 & 0 & 0 & 514 \\
\hline $\begin{array}{l}\text { SIM - Afirmação positiva para o indicador; } \\
\text { NÃO - Afirmação negativa para o indicador; } \\
\text { NSA - Não aplicapilidade ao indicador; } \\
\text { VALOR ABSOLUTO - Soma das ocorrencias analisadas }\end{array}$ & & & & & & & \\
\hline
\end{tabular}

Fonte: Gretzler (2021).

Com a análise da segunda tabela, os itens referentes aos medicamentos e realização dos procedimentos legais e institucionais, aplicado como fórmula de cálculo a mediana, frequência absoluta e percentual total, mostraram que o primeiro indicador da tabela, referente à posologia dos medicamentos, foi de 72,68\% com uma média de 142 em conformidade, porém $24,32 \%$ e a média de 66 não apresentavam conformidade, totalizando um valor absoluto de 3.323 itens. Em média cerca de 50\% dos erros de medicamentos são no momento da solicitação, via ou dose errônea, e por média de 30 a $70 \%$ desses erros são visualizados e solucionados pelo profissional farmacêutico junto à equipe médica (Da Silva, 2020).

O segundo indicador é referente às vias de administração dos medicamentos, sendo observado um valor total de 99,23\% itens de acordo com a via de administração correta, com uma média de 200 e com um valor absoluto de 3.656 itens analisados. Avaliando o terceiro indicador, via de administração está de acordo com a forma farmacêutica escolhida, em 99,97\% itens estavam em conformidade, e apenas $0,03 \%$ dos itens estavam diferentes do recomendado pela bula do fabricante.

A Denominação Comum Brasileira, ou seja, o nome do medicamento pelo seu princípio ativo, foi usado em 76,61\% e cerca de 25,11\% apresentavam inconformidades. O Art. $3^{\circ}$ O Anexo da Resolução RDC n ${ }^{\circ}$ 17, de 2 de março de 2007, passa a vigorar em hospitais públicos, tornando-se obrigatório a utilização do DCB, e em casos específicos a Denominação Comum Internacional (DCI) (Brasil, 2007).

Analisando o aprazamento dos medicamentos observa-se que $63,71 \%$ estavam corretos e cerca de $31,06 \%$ apresentaram inconformidades, sendo grande parte por falta de informações. Corroborando com essa consideração, Jacobsen 
(2015) considera a falta de descrição sobre o aprazamento um erro grave pois o prescritor deve saber em qual frequência será administrado esses medicamentos.

No indicador a forma de diluição está correta, foi observado um total de $63,56 \%$ itens estavam com a forma de diluição correta, entretanto cerca de 36,46\% dos itens analisados não se aplicavam. No indicador está descrito a forma de diluição, constatou-se que 34,55\% dos itens estavam em conformidade, 2,98\% apresentavam inconformidade e 32,01\% dos itens analisados são classificados como não se aplicam, pois são fármacos já prontos para administração.

Já no indicador velocidade de infusão foi identificado que 19,19\% dos itens prescritos estavam em conformidade, cerca de 158 itens não se aplicavam, pois, os medicamentos estavam a critério médico e sem aprazamento, e cerca de 5,5\% dos itens estavam em não conformidade. Neris (2011) relatou em sua dissertação que em 78,20\% de sua amostra, ocorreu a omissão na informação de velocidade de infusão. Segundo Coren-SP (2014) a velocidade de infusão está correlacionada a diversas reações adversas sendo a mais conhecida a síndrome do homem vermelho, que ocorre devido a rápida infusão de vancomicina, sendo de extrema importância relatar a velocidade de infusão seguindo as informações científicas para definir a velocidade, dando prioridade para as informações do fabricante do medicamento.

Durante toda pesquisa não foi identificado nenhum dado sobre medicamentos de uso contínuo. Já no indicador medicamento se necessário, foram identificados 99,18 \% itens com um média de 30 e uma frequência absoluta de 48. Segundo Neri (2011) esse termo é utilizado para que a medicação seja administrada quando for necessário. Nestes casos é crucial que venha prescrito pelo menos o mínimo e máximo da droga e a frequência da administração, além da necessidade de estar descrito o diluente e velocidade de infusão, visando assim evitar erros.

Na pesquisa realizada por Pelliciotti (2010), dezoito profissionais em saúde relataram ter cometido erros no mês que antecedeu a pesquisa, e que somente $61,1 \%$ dos erros foram notificados, com uma média de $67,8 \%$ de que os erros foram realizados no momento da administração do fármaco.

Apesar dos erros serem classificados como evitáveis, não é possível eliminar de forma definitiva da rotina de unidades hospitalares, principalmente em UTI, devido a dinâmica do setor, para tanto, é necessário que o profissional em saúde seja extremamente criterioso e que a equipe multiprofissional esteja atuante de forma íntegra para garantir assim uma melhor assistência e diminuir os erros relacionados aos medicamentos.

\section{Considerações Finais}

Sabemos que o tema cultura de segurança do paciente é um desafio mundial, e que o cuidado seguro resulta de ações corretas dos profissionais de saúde, de processos e sistemas adequados. Infelizmente os erros de prescrições se tornaram comum atualmente, evidenciada como risco a saúde e a vida dos pacientes, sendo necessária a criação de barreiras para diminuírem esses riscos.

Uma das principais barreiras é a comunicação entre a equipe multiprofissional no cuidado ao paciente e a prescrição médica. Deve ser realizada com responsabilidade e conhecimentos técnicos, alinhada aos protocolos assistenciais instituídos, além disso, vale ressaltar que a educação continuada a todos os profissionais da área da saúde é de extrema necessidade visando assim orientá-los na execução da forma correta para minimizar erros.

Outra barreira importante é a atuação do profissional farmacêutico hospitalar e clínico, que pode identificar potenciais erros na prescrição e orientar quanto ao uso correto dos medicamentos, visando assim diminuir possíveis riscos à saúde dos pacientes.

Com base nos resultados obtidos, confirmam-se vários estudos de que o termo evento adverso pode ocorrer não basicamente pela doença de base em si, mas sim pela alta probabilidade de erros de medicação entre outros, devido processos e barreiras considerados inseguros. Este trabalho serve de primícia para que outros mais venham com objetivo incessante da 
busca pelo atendimento seguro além de um compromisso ético, de busca amplamente disseminada nas organizações de saúde, alcançando a qualidade em todos os seus aspectos.

\section{Referências}

Abreu, F. G. S. D. (2013). Erros de Medicação: Avaliação da Prescrição e Percepção dos Profissionais de Enfermagem. Trabalho na conclusão de curso (TCC) na graduação de enfermagem. Universidade de Brasília. Faculdade de Ceilândia.

Alvim, B. A. (2015). Importância da implantação e desenvolvimento da reconciliação medicamentosa em hospitais. Trabalho na conclusão de curso (TCC) em ciências farmacêuticas. Centro Universitário Luterano de Palmas.

Amaro, S., Tavares, E.L. I. A. N. E., Simões, A. P., \& Ribeiro, J. (2017). Prática Profissional Farmacêutica em Unidades Oncológicas: uma reflexão no trabalho. Revista da Jornada de Pós-Graduação e Pesquisa-Congrega Urcamp, 196-217.

Brasil. Ministério da Saúde. Documento de referência para o Programa Nacional de Segurança do Paciente / Ministério da Saúde; Fundação Oswaldo Cruz; Agência Nacional de Vigilância Sanitária. Ministério da Saúde, 2014.

Brasil. Ministério da Saúde. RESOLUÇÃO-RDC No 17, DE 2 DE MARÇO DE 2007. Dispõe sobre o registro de Medicamento Similar e dá outras providências. (2007).

Brasil. Ministério da Saúde. Portaria N 529. Institui o Programa Nacional de Segurança do Paciente (PNSP).2013.

Brasil. Ministério da Saúde Anexo 03: Protocolo de segurança na prescrição, uso e administração de medicamentos. 2017.

Carvalho, V. T., \& Cassiani, S. H. D. B. (2000). Erros na medicação: análise das situações relatadas pelos profissionais de enfermagem. Medicina (Ribeirão Preto), 33(3), 322-330.

CardinaL, L. D. S. M., \& Fernandes, C. S. (2014). Intervenção farmacêutica no processo da validação da prescrição médica. Revista Brasileira de Farmácia Hospitalar e Serviços de Saúde, 5(2).

Costa, D. A. Segurança do Paciente. Martinari.2017

Coren- SP, Conselho Regional de Enfermagem. A qual profissional compete determinar a velocidade da infusão de medicamento injetável. COREN,2014.

Couto, R. C., Pedrosa, T. M. G., Roberto, B. A. D., \& Daibert, P. B. (2017). Anuário da segurança assistencial hospitalar no Brasil. Observatório do cuidado.

CRE-SP, Conselho Regional de Enfermagem. Uso seguro de medicamentos: guia para preparo, administração e monitoramento. COREN, 2017.

Cunha, S. R. F. (2018). O papel do Farmacêutico na reconciliação terapêutica. Repositório Institucional da Universidade Fernando Pessoa.

Da Silva, G. C. A., Pascoa, H., \& Gama, A. R. (2020). Riscos e problemas relacionados aos erros de prescrições médicas: uma revisão bibliográfica. Saúde \& Ciência em ação, 6(2), 134-148.

De Lima, A. P. D., Rios, A. D., Borges, J. S. S. S., Ferreira, J. M., \& Rute, R. (2013). Consulta farmacêutica e análise de problemas relacionados à medicação em um hospital da regional oeste do estado de Goiás. Revista Eletrônica Faculdade Montes Belos, 6(1).

Jacobsen, T. F., Mussi, M. M., \& Silveira, M. P. T. (2015). Análise de erros de prescrição em um hospital da região sul do Brasil. Revista brasileira de farmácia hospitalar e serviços de saúde, 6(3).

Marquis, B. L., \& Huston, C. J. (2015). Administração e liderança em enfermagem: teoria e prática. Artmed editora.

Melo, D. O. D., \& Castro, L. L. C. D. (2017). A contribuição do farmacêutico para a promoção do acesso e uso racional de medicamentos essenciais no SUS. Ciência \& Saúde Coletiva, 22, 235-244.

Nacul, F., Japiassú, A., \& Salluh, J. (2009). Manual de medicina intensiva 1a edição. Elsevier Brasil.

Néri, E. D. R., Gadêlha, P. G. C., Maia, S. G., Pereira, A. G. D. S., Almeida, P. C. D., Rodrigues, C. R. M., \& Fonteles, M. M. D. F. (2011). Erros de prescrição de medicamentos em um hospital brasileiro. Revista da Associação Médica Brasileira, 57, 306-314.

Pena, M. M. (2015). Ocorrência de eventos adversos e sua relação com o fator comunicação em um hospital universitário. Doctoral dissertation, Universidade de São Paulo.

Pires A. O. M, Ferreira M. B.G, Nascimento K. G, Felix M. M. S, Pires P. S, Barbosa M. H. (2017). Elaboration and Validation of the Medication Prescription Safety Checklist. Rev. Latino-Am. Enfermagem.

Pelliciotti, J. D. S. S., \& Kimura, M. (2010). Erros de medicação e qualidade de vida relacionada à saúde de profissionais de enfermagem em unidades de terapia intensiva. Revista Latino-Americana de Enfermagem, 18, 1062-1069.

Tania, A. A., Mário, B. R., Hessem, M. N., Maria, A. P. M. (2010). Fármacia Hospitalar.

Toffoletto, M. C., \& Padilha, K. G. (2006). Conseqüências dos erros de medicação em unidades de terapia intensiva e semi-intensiva. Revista da Escola de Enfermagem da USP, 40, 247-252. 